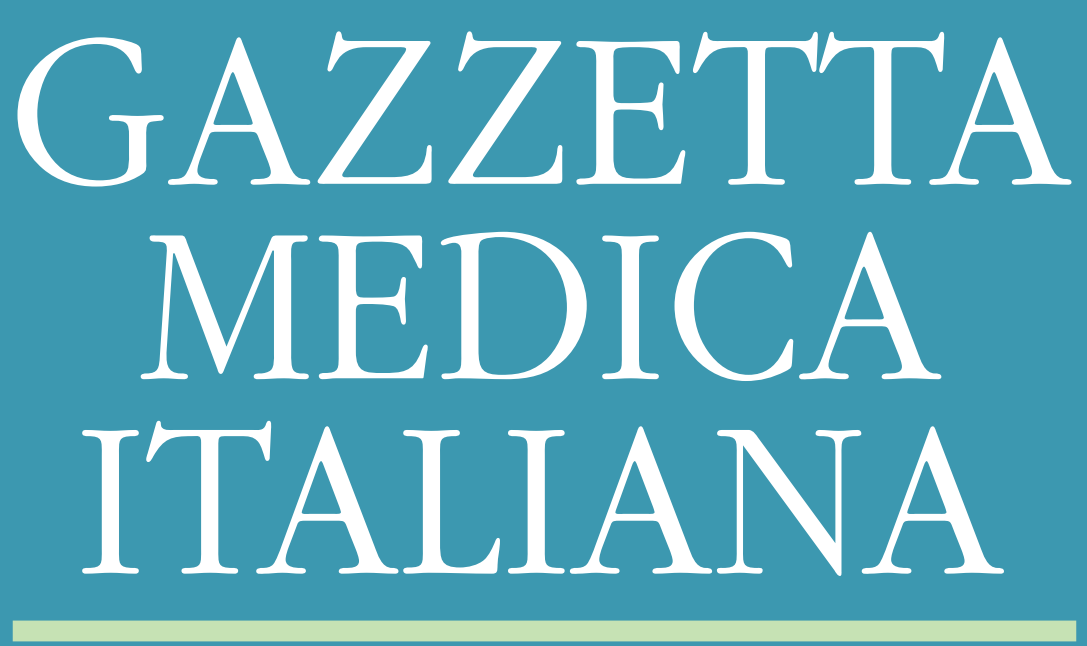

ARCHIVIO PER LE SCIENZE MEDICHE

VOL. $178 \cdot$ No. 10

OCTOBER 2019

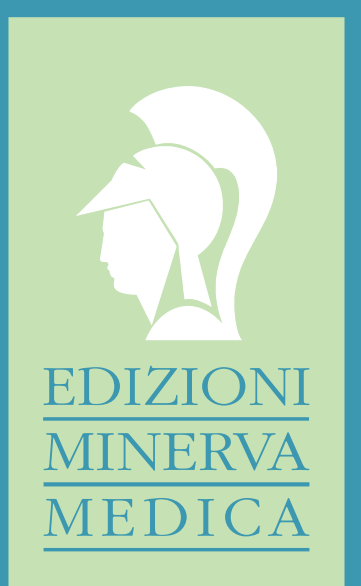




\title{
Sospensione laterale del prolasso degli organi pelvici con protesi in laparoscopia POP-LLS: modifica della tecnica di Dubuisson
}

\author{
Nardo TOLA 1, Fabio MEDAS 2, Maurizio ARRAS $2 *$
}

1Department of Gynecologic Surgery, Sant'Elena Nursing Home, Quartu Sant'Elena, Cagliari, Italy; 2Department of General Surgery, Sant'Elena Nursing Home, Quartu Sant'Elena, Cagliari, Italy

*Corresponding author: Maurizio Arras, Department of General Surgery, Sant'Elena Nursing Home, via Giuseppe Brotzu 6, 09121

Cagliari, Italy. E-mail: maurizio_arras@hotmail.com

\section{R I A S S UN T O}

OBIETTIVO: Valutare i risultati con follow-up di 18.6 mesi della tecnica chirurgica di sospensione laterale del prolasso degli organi pelvici per via laparoscopica (POP-LLS), mediante l'utilizzo di protesi in polipropilene.

METODI: Questo studio retrospettivo è stato eseguito su pazienti di sesso femminile sottoposte ad intervento chirurgico per isterocele, cistocele e/o rettocele sintomatico tra giugno 2013 ed ottobre 2015 nell'Unità ginecologica della Clinica di Sant'Elena a Quartu Sant'Elena, Cagliari, Sardegna (Italia). La tecnica chirurgica di POP-LLS consiste di quattro fasi: la prima fase prevede la sospensione laterale della volta vaginale e dell'utero con una rete in polipropilene posta nel fornice vaginale come fascia trasversale. La seconda consiste in un tunnel retro-peritoneale, utilizzando il trocar e le pinze atraumatiche, dalla parete addominale per raggiungere la plica vescico-uterina precedentemente preparata. Passaggio dell'ala laterale della protesi attraverso il tunnel fino alla sua esternalizzazione cutanea. Questo è ripetuto per entrambe le ali a destra e sinistra. La terza fase prevede il posizionamento senza tensione e la "fissazione" della protesi alla fascia del muscolo addominale trasversale utilizzando vicryl 2.00 (sospensione laterale transparietale). La quarta fase coinvolge, quando indicato, la vaginoplastica posteriore con rettopessi e miorrafia del muscolo elevatore dell'ano.

RISULTATI: Nessun caso (su 100 pazienti in totale), di recidiva del prolasso del compartimento anteriore o centrale si è verificato in nessuno dei pazienti sottoposti a POP-LLS e non si sono verificati casi di erosione o estrusione della protesi. Si è evidenziata la presenza nel postoperatorio di rettocele ex novo in 4 pazienti $(4 \%)$ dei casi. Si è osservata una risoluzione o un miglioramento della SUI in 31 casi su 42 (73,8\%). È stato riscontrato un miglioramento dell'incontinenza da urgenza in 31 casi su 31 donne $(100 \%)$.

CONCLUSIONI: La procedura chirurgica POP-LLS presenta risultati promettenti. Sarà importante tuttavia, valutare tale tecnica su un più ampio numero di pazienti e con follow-up di almeno 3-5 anni per estendere i risultati qui riportati sull'esito di POP-LLS.

\section{A B S T R A C T}

PELVIC ORGANS PROLAPSE LAPAROSCOPY LATERAL SUSPENSION WITH MESH: MODIFICATION OF THE DUBUISSON TECHNIQUE

BACKGROUND: The laparoscopic procedure is proposed as an alternative, minimally invasive technique to restore normal anatomy, improve symptoms in patients with POP (pelvic organ prolapse). We wanted to evaluate the results with a follow-up of 18.6 months of the surgical technique of lateral suspension of pelvic organ prolapse by laparoscopy (POP-LLS), using polypropylene mesh.

METHODS: This retrospective study was performed on female patients undergoing surgery for hysterocele, cystocele and/or symptomatic rectocele between June 2013 and October 2015 in the gynecological unit of the Sant'Elena Clinic in Quartu Sant'Elena, Cagliari, Sardinia (Italy).

RESULTS: No cases (out of 100 patients in total) of relapse of the prolapse of the anterior or central compartment occurred in any of the patients undergoing POP-LLS and there were no cases of erosion or extrusion of the prosthesis. The postoperative presence of rectocele ex novo in 4 patients $(4 \%)$ of cases was highlighted. A resolution or improvement of the SUI was observed in 31 cases out of $42(73.8 \%)$. An improvement in urgency incontinence was found in 31 cases out of 31 women $(100 \%)$. 
CONCLUSIONS: The POP-LLS surgical procedure presents promising results. However, it will be important to evaluate this technique on a larger number of patients and with a follow-up of at least 3-5 years to extend the results reported here on the outcome of POP-LLS.

(Cite this article as: Tola N, Medas F, Arras M. Sospensione laterale del prolasso degli organi pelvici con protesi in laparoscopia POP-LLS: modifica della tecnica di Dubuisson. Gazz Med Ital - Arch Sci Med 2019;178:821-8. DOI: 10.23736/ S0393-3660.18.03971-2)

KEY WORDS: Pelvic organ prolapse; Laparoscopy; Uterus.

$\mathrm{I}_{\mathrm{v}}^{\mathrm{i}}$ termine prolasso degli organi pelvici (POP) viene utilizzato per definire a un ampio spettro di modificazioni che si verificano nella normale anatomia pelvica femminile. Queste possono comprendere un modesto descensus della parete vaginale fino al completo prolasso dai genitali esterni. ${ }^{1}$

L'intervento oggi maggiormente eseguito per trattare il prolasso urogenitale femminile è la colpoisterectomia seguita dalla sospensione dell'apice vaginale secondo McCall, ${ }^{2}$ spesso associata a cisto-rettopessi fasciale.

L'approccio laparoscopico, vede la colposacropessia come "gold standard" per il trattamento del prolasso urogenitale. ${ }^{3}$ In particolare la Cochrane review pubblicata nel 2013 conclude che "la colposcaropessia ha esiti superiori ad una varietà di procedure chirurgiche vaginali tra cui la colpopexy al sacrospinoso, colpopesxy uterosacrale e alle tecniche con utilizzo di reti transvaginali", sottintendendo che tutte le forme di colposacropessia sono superiori a tutte le altre opzioni chirurgiche per il trattamento del prolasso apicale. ${ }^{4}$

La tecnica analizzata ed illustrata nel presente studio trae ispirazione dal lavoro clinico e chirurgico del Prof. JB Dubuisson, la cui prima pubblicazione sull'argomento risale al 19985,6 e che a sua volta si basa sul lavoro di Kapandji, il quale ha eseguito la stessa tecnica per via laparotomica. ${ }^{7}$

La nostra tecnica chirurgica è una modifica di quella originariamente proposta da Dubuisson et al. 5

La procedura laparoscopica è proposta come tecnica alternativa, minimamente invasiva, per ristabilire l'anatomia normale, migliorare i sintomi e ristabilire le normali funzioni urinarie, intestinali e sessuali. L'obiettivo di questo studio retrospettivo è di valutare $\mathrm{i}$ risultati ottenuti nella coorte di 100 pazienti sottoposte a trattamento del prolasso degli organi pelvici femminili con follow-up di 18,6 mesi.

\section{Materiali e metodi}

Cento pazienti sono state trattate tra il 2013 e il 2015 utilizzando la tecnica di Dubuisson modificata di sospensione laterale del prolasso degli organi pelvici con protesi in laparoscopia (POPLLS).

Nel presente lavoro, illustriamo i risultati ottenuti dal trattamento chirurgico di questi pazienti affetti da prolasso urogenitale di varie entità e gradi e / o da incontinenza urinaria da stress e / o da urgenza minzionale.

\section{Gruppo pazienti}

Da giugno 2013 ad ottobre 2015 (follow-up medio: $18,6 \pm 7,5$ mesi), 100 pazienti affette da prolasso degli organi pelvici (POP), sono state sottoposte a chirurgia di sospensione laterale laparoscopica con rete protesica (POP-LLS). Tutti gli interventi sono stati eseguiti dallo stesso chirurgo (N.T.).

Le pazienti sono state informate sulle caratteristiche dell'operazione mediante colloquio con i medici della equipe chirurgica e hanno fornito a questi il loro consenso informato scritto.

Le controindicazioni associate all'operazione sono le stesse proprie della laparoscopia in generale; la principale controindicazione è rappresentata dalle complicazioni cardio-respiratorie.

I dati anamnestici per tutti le pazienti sono stati inseriti in una banca dati appositamente creata dove ciascun caso clinico è stato registrato in base al grado di prolasso urogenitale secondo la classificazione di Baden-Walker, ${ }^{8}$ al grado di incontinenza urinaria da sforzo secondo la classificazione di Ingelman-Sundberg. ${ }^{9}$

Il successo della procedura chirurgica è stato stabilito sulla base della restitutio ad integrum anatomica e funzionale e della riduzione del 
grado di prolasso secondo la classificazione di Baden-Walker. ${ }^{8}$

I pazienti sono stati riesaminati dalla nostra equipe 1, 3 e 6 mesi dopo l'intervento e dopo 3 anni contattati ed intervistati telefonicamente per quanto riguarda i seguenti aspetti clinici: senso di pesantezza vulvo-perineale, dolore pelvico, pollachiuria, disuria, incontinenza urinaria da stress ed incontinenza urinaria da urgenza, costipazione, dispareunia (Tabella I).

I seguenti dati sono stati registrati in un database elettronico tenendo conto di caratteristiche demografiche incluso BMI e parità (Tabella II); dati preoperatori, dati relativi all'intervento chirurgico stesso, eventuali complicanze postoperatorie e dati relativi al risultato chirurgico.

\section{Studio preoperatorio}

Tutte le pazienti sono state precedentemente registrate in un database elettronico contenente anamnesi, esami del sangue di routine, esame clinico, ecografia pelvica transvaginale con valutazione dei compartimenti anteriore, centrale e posteriore secondo la classificazione HWS (Baden-Walker Half Way System) (Tabella III) ${ }^{6}$ e la

TABELlA I.-Caratteristiche preoperatorie.

\begin{tabular}{lc}
\hline & (POP-LLS) (N.=100) \\
\hline Isterocele & $21(21 \%)$ \\
Grado 1-2 & $66(66 \%)$ \\
Grado 3-4 & \\
Cistocele & $26(26 \%)$ \\
Grado 1-2 & $62(62 \%)$ \\
Grado 3-4 & \\
Rettocele & $11(11 \%)$ \\
Grado 1 & $10(10 \%)$ \\
Grado 2 & $11(11 \%)$ \\
Grado 3 & \\
IUS (incontinenza urinaria da sforzo) & $11(11 \%)$ \\
Grado 1 & $29(29 \%)$ \\
Grado 2 & $2(2 \%)$ \\
Grado 3 & $31(31 \%)$ \\
Urge Inc. (incontinenza da urgenza) & \\
\hline
\end{tabular}

TABELla II.-Dati demografici.

\begin{tabular}{lc}
\hline & (POP-LLS) \\
\hline N. di pazienti & 100 \\
Età (anni) & $62.9 \pm 9.8$ \\
Parità & $2.6 \pm 1.22$ \\
BMI $\left(\mathrm{Kg} / \mathrm{m}^{2}\right)$ & $25.4 \pm 3.2$ \\
\hline
\end{tabular}

TABELLA III.-Classificazione Baden and Walker ${ }^{6}$

\begin{tabular}{ll}
\hline Grado 0 & No descensus \\
Grado 1 & Descensus tra spina ischiatica ed imene \\
Grado 2 & Descensus quasi all'imene \\
Grado 3 & Descensus oltre l'imene \\
\hline
\end{tabular}

TABELla IV.-Classificazione Ingelman-Sundberg. ${ }^{10}$

\begin{tabular}{ll}
\hline Grado 1 & Solo come conseguenza di tosse spontanea \\
Grado 2 & Quando si cammina o si salgono le scale \\
Grado 3 & Avviene per minimi movimenti \\
\hline
\end{tabular}

valutazione dell'incontinenza urinaria secondo la classificazione di Ingelman-Sundberg (Tabella IV). 9,10

\section{Tecnica operatoria}

Le pazienti sono state sottoposte a POP-LLS (sospensione laterale del prolasso degli organi pelvici con protesi in laparoscopia).

Il trattamento chirurgico per il prolasso urogenitale femminile è stato effettuato con laparoscopia in anestesia generale ed intubazione endotracheale. Tranne che in un piccolo numero di casi, le nostre tecniche consentono di conservare l'utero se privo di qualsiasi patologia.

La procedura chirurgica consiste di tre fasi, più una quarta se presente rettocele di grado 1 oppure oltre.

\section{Fase 1}

Incisione sovrapubica mediana ed inserimento del trocar da $12 \mathrm{~mm}$. Incisione della cute a destra e sinistra $2 \mathrm{~cm}$ posteriormente, $6-8 \mathrm{~cm}$ sopra la spina iliaca anteriore-superiore.

Inserimento di 2 trocar da $5 \mathrm{~mm}$. Esposizione della parete vaginale e del fornice anteriore utilizzando la valva all'interno della vagina. Incisione e separazione della plica vescico-uterina verso il basso, esponendo un'area della fascia pubocervicale corrispondente al grado di cistocele.

Fissazione della porzione centrale della rete in polipropilene (dotata di due bracci di trazione laterali) alla parete anteriore della vagina e dell' $i$ stmo con 4 suture Ti-Cron " 0 ". Il primo punto di ancoraggio viene dato in vicryl, rinforzando così la fascia pubocervicale, creando una sospensione per la vagina, appiattendo ed eliminando così il cistocele (Figura 1). 


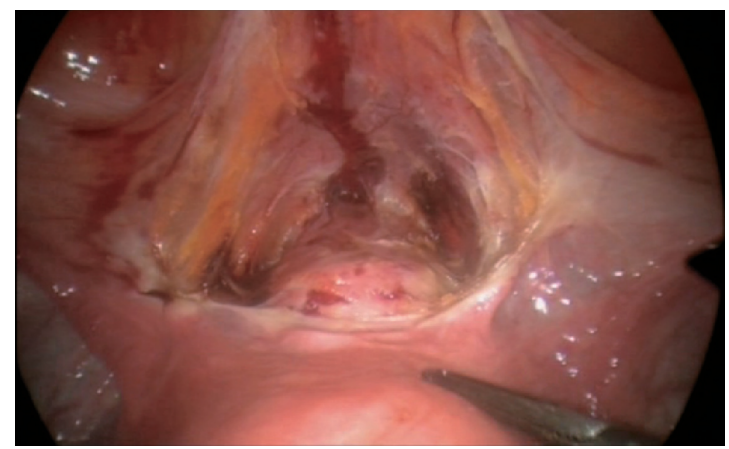

Figura 1.-Dall'immagine si evince lo scollamento della plica vescico-uterina e l'esposizione delle spazio necessario alla protesi che verrà agganciata alla fascia pubo-cervicale anteriore e al fornice vaginale.

\section{Fase 2}

Estrazione parziale del trocar laterale e suo inserimento nel piano preperitoneale. Tunnellizzazione retroperitoneale, utilizzando il trocar e le pinze atraumatiche, della parete addominale per raggiungere la plica vescico-uterina. Passaggio dell'ala destra della rete attraverso il tunnel fino all'esternalizzazione cutanea. Questo è ripetuto per l'ala sinistra.

Peritonizzazione con Monocryl 0 che attraversa il peritoneo prevescicale, il legamento rotondo destro (la cui lunghezza assoluta verrà ridotta), la superficie anteriore dell'utero (che si anteriorizzerà fino alla sua posizione fisiologica di antiversione), il legamento rotondo sinistro e ancora il peritoneo. Alla fine, nessuna porzione della protesi è esposta alla cavità pelvica (Figura 2).

\section{Fase 3}

Posizionamento tension-free e fissazione della protesi alla fascia del muscolo addominale trasverso mediante vicryl 2.00 (Figura 3).

\section{Fase 4}

In presenza di Rettocele di III-IV grado secondo HWS, la quarta fase coinvolge la vaginoplastica posteriore con rettopessi che coinvolge la fascia presacrale e la miorrafia dei muscoli elevatori dell'ano.

Le mesh preconfigurate qui utilizzate prodotte dalla ditta ANGIOLOGICA B.M. SRL (via Giovanni XXIII, 4 - 27028 - S. Martino Siccomario
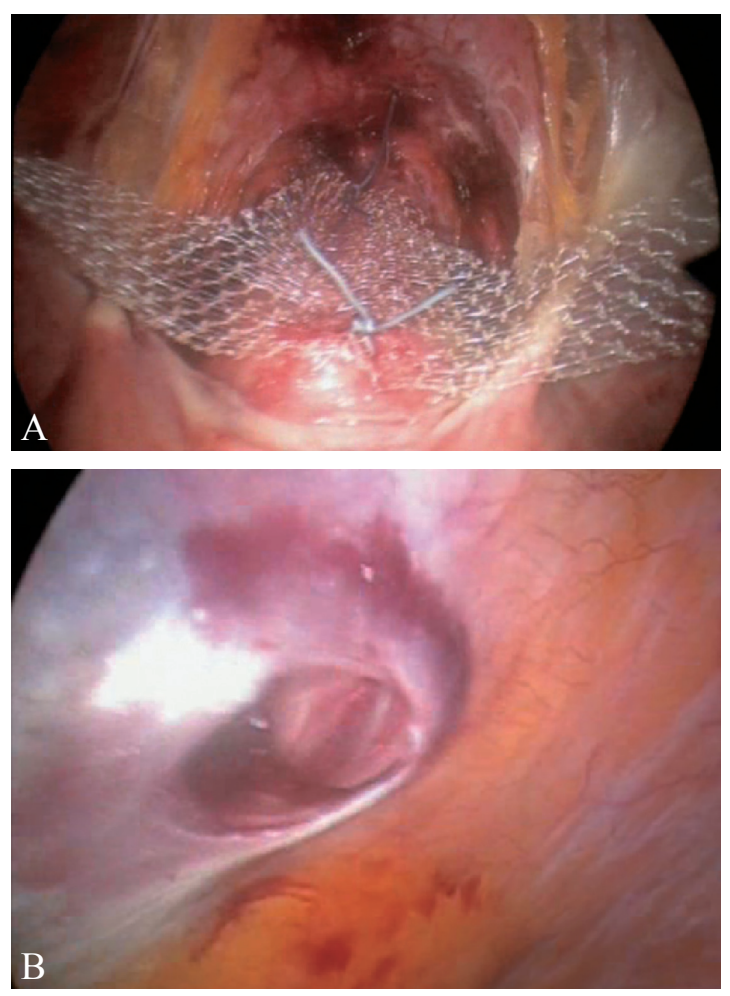

Figura 2.-A) La parte centrale della protesi viene adagiata nella tasca creata scollando la plica vescico-uterina. Sono stati posizionati alcuni punti di sutura. B) Il trocar di destra sta per essere inserito nel retro peritoneo.

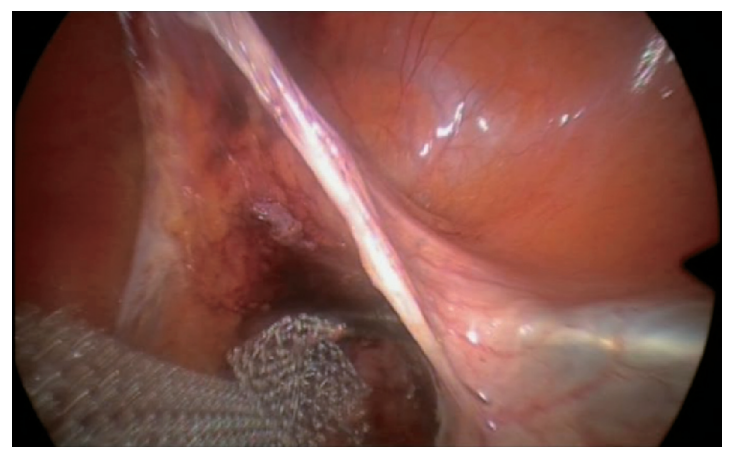

Figura 3.- La pinza di Manes afferra il braccio destro della protesi per passare nel retroperitoneo.

- Pavia Italy), e realizzate in polipropilene, possono essere ulteriormente rimodellate $a d$ hoc in base alle esigenze di ciascun paziente. ${ }^{11}$ La protesi viene fornita con una configurazione a $\mathrm{T}$ o a forma di croce per la correzione rispettivamente dell'isterocistocele o nel prolasso della volta vaginale. Le maglie in polipropilene corrispondono 
al tipo 1, secondo la classificazione della protesi chirurgica, ${ }^{12}$ che soddisfa i requisiti di cui sopra.

La protesi consiste di una porzione centrale che può essere modellata su ogni singolo paziente in base alle esigenze (la dimensione del cistocele) e misura $3 \mathrm{~cm} \times 4 \mathrm{~cm}$. Ha due bracci o ali di trazione laterali lunghe circa $25 \mathrm{~cm}$ e larghi $27 \mathrm{~mm}$. La protesi viene inserita arrotolata dal trocar centrale sovrapubico di $12 \mathrm{~mm}$.

Nei casi di patologia benigna dell'utero, e in assenza di qualsiasi patologia della cervice accertata preventivamente mediante uno screening cervicale (Pap test), ecografia pelvica transvaginale e, quando indicato isteroscopia, può essere eseguita un'isterectomia preliminare; a nostro avviso, l'isterectomia sopracervicale è la soluzione migliore per mantenere la stabilità pelvica, poiché l'isterectomia sopracervicale offre un eccellente supporto alla protesi con un minor rischio di erosione della stessa.

\section{Analisi statistica}

I dati sono stati analizzati utilizzando la versione del software MedCalc ${ }^{\circledR}$. 12.7.0.0.

\section{Risultati}

Il $94 \%$ delle pazienti (94/100 totali), hanno riportato un livello medio-alto di soddisfazione postoperatoria.

\section{Risultato chirurgico}

Nella Tabella V sono indicati gli esiti chirurgici. La Tabella VI e la Tabella VII, mostrano come le pazienti siano state ricoverate in ospedale per

TABELLA V.-Risultato post chirurgico.

\begin{tabular}{lc}
\hline & $\begin{array}{c}\text { (POP-LLS) } \\
\text { (N.=100) }\end{array}$ \\
\hline Prolasso o recidiva della cupola vaginale. & 0 \\
Comparsa di Rettocele & $4 / 32(12.5 \%)$ \\
IUS de novo & $3 / 58(5.2 \%)$ \\
Nessun cambiamento o peggioramento della & $11 / 42(26.2 \%)$ \\
$\quad$ IUS & \\
Risoluzione o miglioramento della IUS & $31 / 42(73.8 \%)$ \\
Urge Inc de novo & $13 / 87(13 \%)$ \\
Risoluzione della Urge Inc & $31 / 31(100 \%)$ \\
Alterazione della sensibilità cutanea & $4(4 \%)$ \\
$\quad$ nell'area di innervaizone del nervo Genito- & \\
Femorale. & \\
\hline
\end{tabular}

circa 48 ore ed hanno urinato immediatamente dopo la rimozione del catetere vescicale avvenuta la stessa sera dopo intervento chirurgico. Le complicanze sono riportate nella Tabella VIII ed hanno riguardato una lesione accidentale della parete vescicale durante lo scollamento della plica vescico-uterina, un caso di estrusione in vescica del filo Ti-Cron ed infine 4 casi di alterazione della sensibilità cutanea con dolore nel territorio innervato dal nervo Genito-Femorale.

\section{Discussione}

I risultati emersi da questo studio sono incoraggianti ed in linea con quelli presentati da Dubuisson nel 2011,13 i quali riportavano un tasso di successo dell' $83,8 \%$. Bisogna precisare a tale proposito che la tecnica chirurgica riportata in questo studio prevede la modifica di quella proposta dallo stesso Dubuisson con l'ancoraggio dei bracci laterali della protesi alla fascia del muscolo addominale trasverso mediante Vicryl 2.0. Infatti ad oggi non si sono registrati casi di recidiva (cioè a partire dallo stadio 2 di Baden Wal-

TABELla VI.-Giorni di degenza.

\begin{tabular}{lc}
\hline Dimissione dopo chirurgia & POP-LLS $(\mathrm{N} .=100)$ \\
\hline 2 giorni & 100 \\
3 giorni & 0 \\
4 giorni & 0 \\
$>5$ giorni & 0 \\
\hline
\end{tabular}

TABella VII.-Giorni in cui la diuresi si è verificata spontaneamente dopo chirurgia.

\begin{tabular}{lc}
\hline Diuresi spontanea dopo chirurgia giorni: & POP-LLS $(\mathrm{N} .=100)$ \\
\hline 1 giorno & 100 \\
2 giorni & 0 \\
3 giorni & 0 \\
\hline
\end{tabular}

TABELLA VIII.-Complicanze post chirurgiche.

Complicanze Group A (N.=100)

Danno ad anse intestinali 0

Trasfusioni di sangue o di emoderivati 0

Lesioni alla vescica

Estrusione di filo TI CRON in vescica $\quad 1$

Ematoma para uretrale $\quad 0$

Lesioni ureterali

0
0
1
1
0
0


ker), mentre Dubuisson registra (nel lavoro citato sopra), un tasso di recidive del $13,76 \%$. Nel presente studio abbiamo osservato che tutte le pazienti $(100 / 100$ casi totali, $100 \%)$, hanno presentato dolore nel punto di ancoraggio dei bracci laterali della protesi alla fascia del muscolo trasverso dell'addome. Tale dolore, di intensità variabile (da 3 a 6 della scala VAS), si è protratto da subito dopo l'intervento fino a 45-60 giorni del post operatorio in alcuni casi per poi scomparire.

Per quanto riguarda l'urgenza minzionale, 31 pazienti nella nostra coorte che avevano riferito di soffrire del problema prima di sottoporsi a POP-LLS, hanno mostrato una completa risoluzione rispetto alla condizione preoperatoria; mentre 13 pazienti su 100 (13\%), delle pazienti ha riferito urgenza minzionale de novo insorta nel postoperatorio.

Come è noto, molte teorie sono state poste fin dall'inizio del secolo scorso rivolte a spiegare il fenomeno dell'incontinenza urinaria. Tra le teorie più importanti ricordiamo qui quella di Kelly (1914), ${ }^{14}$ Richardson (1976); 15 Papa Petros. ${ }^{16,} 17$ Tuttavia, sembrerebbe che gli effetti positivi (miglioramento, risoluzione) della POP-LLS sull'incontinenza urinaria possano essere spiegati dalla teoria di Enhorning formulata nel 1961.18

Prima di effettuare l'intervento di POP-LLS, è importante esaminare le anomalie uterine più significative, come peraltro suggerito da Gutman e Maher, tra cui: fibromi uterini, adenomiosi, patologia della cervice uterina e dell'endometrio, sanguinamento uterino anomalo in epoca postmenopausale, terapia con tamoxifene e predisposizione familiare a tumori. ${ }^{10}$

Per ciò che riguarda il fenomeno dell'erosione della protesi in polipropilene, un recente studio caso-controllo di Dällenbach sull'incidenza e sui fattori di rischio per l'erosione della protesi dopo POP-LLS, ${ }^{19}$ ha concluso che la rete protesica è sicura.

Nel presente studio non si sono verificati casi di recidiva del prolasso del compartimento anteriore (isterocistocele) e ciò è probabilmente dovuto al nostro ancoraggio della protesi alla fascia dei muscoli obliqui addominali che, secondo la letteratura, non è praticato da nessun altro allo stato attuale. Ad esempio, uno studio del $2011 \mathrm{di}$ Dubuisson, ${ }^{13}$ che presenta il maggior numero di casi di POP-LLS con periodo di follow-up più lungo riportato ad oggi, descrive un tasso di recidiva complessivo del $13,76 \%$ considerando tutti i vari compartimenti.

Nonostante il successo del presente studio e l'assenza di recidive del compartimento anteriore, riportiamo anche il presentarsi de novo di rettocele nel $4 \%$ dei casi ed un peggioramento nel grado di rettocele preesistente nel $4 \%$ dei casi dopo l'intervento.

È molto probabile infatti che dopo la POP-LLS con conseguente anteriorizzazione dell'utero, fino a quel momento in retroversione, le forze vettoriali della pressione endo-addominale agiscano direttamente sul retto, mettendo ancor più in difficoltà e in sovraccarico la fascia endopelvica, così da determinare un locus minoris resistentiae, provocando infine un rettocele de novo o peggiorando una condizione preesistente. Per questi motivi attualmente, anche il rettocele di grado $1^{\circ}$ viene sempre trattato con colpoperineoplastica dopo POP-LLS nella stessa seduta operatoria.

In uno dei suoi studi più recenti eseguito su una serie di 245 pazienti, con un follow-up medio di 7,5 anni, pubblicato nel 2016, ${ }^{20,} 21$ Dubuisson riporta un tasso di successo anatomico pari all' $88,2 \%$ dopo 12 mesi per il compartimento anteriore, uno del $86,1 \%$ per il compartimento apicale, ed uno del $80,8 \%$ per il compartimento posteriore (anche Dubuisson esegue la rettopessi fasciale).

Nel presente studio, abbiamo osservato 4/100 (4\%), casi di dolore accompagnato da alterazione della sensibilità della cute nell'area di competenza del nervo genitofemorale. Associamo questa sintomatologia alla compressione che la protesi può esercitare sul nervo genitofemorale, che attraversa il muscolo ileopsoas, al di sopra del quale la protesi passa nel suo percorso retroperitoneale. È anche interessante notare che questi sintomi di disagio interessavano maggiormente le pazienti molto magre.

È possibile che in queste donne il nervo genitofemorale non sia protetto da un cuscinetto di tessuto adiposo che lo circondi nel suo passaggio attraverso il muscolo ileopsoas, cosa che avviene nelle donne normali o in sovrappeso.

Tutti i casi sono stati comunque trattati per 65 gg con acido lipoico, complesso vitaminico B e palmitoiletanolamide (SuperAla $800 \mathrm{mg}+$ Pel- 
vilen forte $400 \mathrm{mg}$ ), determinando la completa risoluzione dei sintomi.

Analizzando l'effetto positivo della POP-LLS sulla incontinenza urinaria da sforzo (SUI) e sulla urgenza minzionale, i risultati del presente studio sono particolarmente sorprendenti, tanto da migliorare ed in molti casi a risolvere la sintomatologia specifica dopo l'intervento chirurgico. In particolare, 42 pazienti su 100 sottoposte a POPLLS erano affette dalla SUI; 11 di queste hanno presentato SUI di primo grado, 29 SUI di grado 2 e 2 pazienti hanno presentato SUI di grado 3. Dopo chirurgia POP-LLS, 25/100 (25\%) donne hanno riferito la risoluzione dei sintomi di SUI ( 7 che avevano il primo grado e 18 che avevano un SUI di grado 2).

Sei donne hanno dichiarato un miglioramento della condizione, dal grado 2 al grado 1 secondo $i$ criteri di classificazione di Ingelman-Sundberg. ${ }^{9}$

Tre pazienti (3\%) hanno dichiarato nuove manifestazioni di SUI in seguito a POP-LLS.

Il tasso di risoluzione, d'altra parte, era del $59,5 \%$ (25 su 42 colpiti dalla SUI). Il tasso di miglioramento per i pazienti con SUI di grado 2 era del 14\%; quindi un $73,8 \%$ dei pazienti con SUI ha riportato benefici dopo l'intervento per quanto riguarda questi sintomi.

La conservazione dell'utero è diventata sempre più oggetto di attenzione, soprattutto nei casi di giovani donne che desiderano preservare la loro fertilità. Skorupska e collaboratori 22 hanno recentemente pubblicato uno studio in cui hanno analizzato la percentuale di nuovi casi di incontinenza urinaria dopo colpo isterectomia definendo questa pratica come un fattore di rischio per le disfunzioni postoperatorie delle basse vie urinarie e che il livello di rischio dipende dal tipo di isterectomia e dalla causa iniziale che determina la necessità dell'intervento.

Secondo Dubuisson ${ }^{13,} 20$ e Wattiez, ${ }^{23}$ formati in chirurgia laparoscopica, la colposacropessia rappresenta il gold standard per il trattamento del prolasso urogenitale.

Nel 2005, Higgs pubblicò uno studio sui risultati della colposacropessia. ${ }^{24} \mathrm{Su} 140$ casi, riporta un'eccellente sospensione dell'apice vaginale nel $92 \%$ dei casi, tuttavia con un'incidenza di recidive pari al 38\% (di altri compartimenti) e un tasso di chirurgia ripetuta del $16 \%$ entro un periodo di follow-up di 66 mesi. Inoltre, Higgs riporta anche un'incidenza di erosione della rete protesica in prolene del 6\%. La colposacropessia richiede anche operatori esperti altamente qualificati (Wattiez descrive una curva di apprendimento ottimistica con plateau raggiunto dopo circa 24 procedure), ${ }^{25}$ con perfetta conoscenza dell'anatomia pelvica e del retroperitoneo; inoltre, dovrebbe preferibilmente essere eseguito dopo isterectomia totale o sopracervicale.

Nel 2011, Dubuisson ha descritto la POP-LLS come una procedura sicura, con un tasso di complicanze a breve termine (comprese le complicanze minime) nell'ordine del 5,9\%.13 Secondo questo studio, la procedura non comporta il rischio di danni vascolari o nervosi (legati anche alle varianti anatomiche), o rischi che coinvolgono i legamenti, come il legamento largo, poiché il passaggio della protesi avviene sotto i legamenti rotondi. Questo è senza dubbio un vantaggio della POP-LLS rispetto alla colposacropessia laparoscopica dove tali rischi sono sempre presenti. Una complicazione classica di quest'ultima è ad esempio l'estrusione vaginale della protesi, la cui incidenza, secondo la letteratura, è compresa tra lo 0 e il $13 \%$; questo rischio è 5 volte inferiore se si conserva l'utero, praticando cioè l'isterosacropessia [Gutman e Maher]. Nel presente studio comunque non sono stati osservati casi di estrusione o di erosione della rete protesica (followup medio: $18,6 \pm 7,5$ mesi).

\section{Conclusioni}

In sintesi, la sospensione laterale del prolasso degli organi pelvici con protesi in laparoscopia è una tecnica mini-invasiva che tratta efficacemente il prolasso degli organi pelvici femminili del comparto anteriore e centrale e parimenti dell'incontinenza urinaria. Si presenta come una tecnica facilmente riproducibile, conservativa, associata al minor rischio di complicanze e recidive; soprattutto se seguita da colpoplastica posteriore complementare e terapia estrogenica vaginale a basso dosaggio ove possibile. ${ }^{26}$

Sarà importante tuttavia, valutare tale tecnica su un più ampio numero di pazienti e con followup di almeno 3-5 anni per estendere i risultati qui riportati sull'esito di POP-LLS. 


\section{Bibliografia}

1. Pescetto G, De Cecco L, Pecorari D, Ragni N. Ginecologia e Ostetricia. Volume 1. Roma: Società Ed. Universo, Roma; 2009. p. 1-22.

2. Cruikshank SH, Kovac SR. Randomized comparison of three surgical methods used at the time of vaginal hysterectomy to prevent posterior enterocele. Am J Obstet Gynecol 1999;180:859-65.

3. Wattiez A, Mashiach R, Donoso M. Laparoscopic repair of vaginal vault prolapse. Curr Opin Obstet Gynecol 2003;15:315-9.

4. Maher C, Feiner B, Baessler K, Schmid C. Surgical management of pelvic organ prolapse in women. Cochrane Database Syst Rev 2013;4:CD004014.

5. Dubuisson JB, Chapron C. Laparoscopic iliac colpouterine suspension for treatment of genital prolapse using two meshes. A new operative technique. J Gynecol Surg 1998;14:153-9.

6. Dubuisson JB, Chapron C, Fauconnier A, Babaki-Fard K, Dendrinos S. Laparoscopic management of genital prolapse: lateral suspension with two meshes. Gynaecol Endosc 2000;9:3683-9.

7. Kapandji M. [Treatment of urogenital prolapse by colpoisthmo-cystopexy with transverse strip and crossed, multiple layer, ligamento-peritoneal douglasorrhaphy]. Ann Chir 1967;21:321-8. French.

8. Walker T. Surgical repair of vaginal defects. Philadelphia: Lippincott; 1992. p. 14.

9. Ingelman-Sundberg A, Ulmsten U. Surgical treatment of female urinary stress incontinence. Contrib Gynecol Obstet 1983;10:51-69.

10. Gutman R, Maher C. Uterine-preserving POP surgery. Int Urogynecol J Pelvic Floor Dysfunct 2013;24:1803-13.

11. Angiologica Company, Via Giovanni XXIII, 4 - 27028 S. Martino Siccomario Pavia (PV), Italy.

12. Chu CC, Welch L. Characterization of morphologic and mechanical properties of surgical mesh fabrics. J Biomed Mater Res 1985;19:903-16.

13. Dubuisson JB, Eperon I, Jacob S, Dubuisson J, Wenger JM, Dallenbach P, et al. [Laparoscopic repair of pelvic organ prolapse by lateral suspension with mesh: a continuous series of 218 patients]. Gynécol Obstét Fertil 2011;39:127-31. French.
14. Kelly HA, Dumm WM. Urinary incontinence in women, without manifest in-jury to the bladder. Surg Gynecol Obstet 1914;18:444-53.

15. Richardson AC, Lyon JB, Williams NL. A new look at pelvic relaxation. Am J Obstet Gynecol 1976;126:568-73.

16. Petros PE, Ulmsten UI. An integral theory of female urinary incontinence. Experimental and clinical considerations. Acta Obstet Gynecol Scand Suppl 1990;153:7-31.

17. Petros PE, Ulmsten UI. An integral theory and its method for the diagnosis and management of female urinary incontinence. Scand J Urol Nephrol Suppl 1993;153:1-93.

18. Enhorning G. Simultaneous recording of intravesical and intra-urethral pres-sure: a study on urethral closure in normal and stress incontinence women. Acta Chir Scand 1961; suppl. 276:1-68.

19. Dällenbach P, De Oliveira SS, Marras S, Boulvain $\mathrm{M}$. Incidence and risk factors for mesh erosion after laparoscopic repair of pelvic organ prolapse by lateral suspension with mesh. Int Urogynecol J Pelvic Floor Dysfunct 2016;27:1347-55

20. Veit-Rubin N, Dubuisson JB, Lange S, Eperon I, Dubuisson J. Uterus-preserving laparoscopic lateral suspension with mesh for pelvic organ prolapse: a patient-centred outcome report and video of a continuous series of 245 patients. Int Urogynecol J Pelvic Floor Dysfunct 2016;27:491-3.

21. Serati M, Giarenis I, Meschia M, Cardozo L. Role of urodynamics before prolapse surgery. Int Urogynecol J Pelvic Floor Dysfunct 2015;26:165-8.

22. Skorupska KA, Miotła P, Kubik-Komar A, Rechberger E, Adamiak-Godlewska A, Rechberger T. Urinary incontinence after hysterectomy- does type of surgery matter? Ginekol Pol 2016;87:94-7.

23. Gabriel B, Nassif J, Barata S, Wattiez A. Twenty years of laparoscopic sacrocolpopexy: where are we now? Int Urogynecol J Pelvic Floor Dysfunct 2011;22:1165-9.

24. Higgs PJ, Chua HL, Smith AR. Long term review of laparoscopic sacrocolpopexy. BJOG 2005;112:1134-8.

25. Akladios CY, Dautun D, Saussine C, Baldauf JJ, Mathelin C, Wattiez A. Laparoscopic sacrocolpopexy for female genital organ prolapse: establishment of a learning curve. Eur J Obstet Gynecol Reprod Biol 2010;149:218-21.

26. Weber MA, Kleijn MH, Langendam M, Limpens J, Heineman MJ, Roovers JP. Local Oestrogen for Pelvic Floor Disorders: A Systematic Review. PLoS One 2015;10:e0136265.

Conflicts of interest.-The authors certify that there is no conflict of interest with any financial organization regarding the material discussed in the manuscript.

Authors' contributions.-Nardo Tola: conception and design of study, responsible surgeon, data analysis and interpretation; Fabio Medas: statistical analysis, manuscript preparation, data analysis and interpretation; Maurizio Arras: conception and design of study, manuscript preparation, data analysis and interpretation, patient recruitment.

Manuscript accepted: October 2, 2018. - Manuscript received: September 10, 2018 